\title{
Digital Records: A valuable Tool in Orthodontics
}

\author{
Anshul Chaudhry ${ }^{1}$ and Girish Chaudhary ${ }^{2}$ \\ ${ }^{1}$ Associate Professor, Department of Orthodontics, Christian Dental College, CMC, Ludhiana, Punjab, India \\ ${ }^{2}$ Reader, Department of Orthodontics, Baba Jaswant Singh Dental College, Hospital and Research Institute, Chandigarh Road, Ludhiana, \\ Punjab, India \\ *Corresponding Author: Anshul Chaudhry, Associate Professor, Department of Orthodontics, Christian Dental College, CMC, Ludhiana, \\ Punjab, India.
}

Received: August 22, 2019; Published: September 20, 2019

DOI: 10.31080/ASDS.2019.03.0646

The long durations of Fixed orthodontic treatment has always been a matter of concern for the patients. Along with the experience of the clinician, accurate diagnosis and treatment planning plays an important role for the completion of treatment well in time and for the long term stability. For the proper diagnosis, one has to obtain a large amount of information from the different diagnostic tools. With the increasing awareness about the braces, now a days, adults are also opting for the orthodontic treatment. For the complex cases, which involve the multidisciplinary approach, one has to be more cautious about the record keeping and maintenance of the records. It is very difficult to run with the models, photos and radiographs from one department to another. The digitalization of records have solved this cumbersome job of maintaining the records at each and every step, with the introduction of CAD which makes use of certain processing tools to generate the images. New software's have come up to do the model, cephalometric and photographic analysis which otherwise was done manually and took long hours. With the shift of technologies being used to diagnose for the medical purpose to the dental side has eased the job at every step. Three dimensional views can be generated from CT, CBCT and MRI to reconstruct the skeletal and dentofacial regions along with the soft tissue assessment which might be helpful for the surgical cases. So, this transition from models, photos and radiographs to digitalization has been successful because of computers only. It has really helped to obtain a proper diagnosis and in providing a better treatment to the patients, even in the routine.

\section{Volume 3 Issue 10 October 2019 \\ (C) All rights are reserved by Anshul Chaudhry and Girish Chaudhary.}

